\title{
A Case of Pentastomiasis at the Left Maxilla Bone in a Patient with Thyroid Cancer
}

\author{
Eunae Sandra Cho', Seung Wook Jung², Hwi-Dong Jung'2, In Yong Lee³, Tai-Soon Yong ${ }^{3}$, Su Jin Jeong ${ }^{4}$, \\ Hyun Sil Kim ${ }^{1, *}$
}

\begin{abstract}
${ }^{1}$ Department of Oral Pathology, Oral Cancer Research Institute, Yonsei University College of Dentistry, Seoul 03722, Korea; '2Department of Oral and Maxillofacial Surgery, Yonsei University College of Dentistry, Seoul 03722, Korea; ${ }^{3}$ Department of Environmental Medical Biology, Institute of Tropical Medicine \& Arthropods of Medical Importance Bank, Yonsei University College of Medicine, Seoul 03722, Korea; ${ }^{4}$ Department of Internal Medicine and AIDS Research Institute, Yonsei University College of Medicine, Seoul 03722, Korea
\end{abstract}

\begin{abstract}
Pentastomiasis, a zoonotic parasite infection, is typically found in the respiratory tract and viscera of the host, including humans. Here, we report for the first time an extremely rare case of intraosseous pentastomiasis in the human maxilla suffering from medication related osteonecrosis of the jaw (MRONJ). A 55-year-old male had continuously visited the hospital for MRONJ which had primarily developed after bisphosphonate and anti-neoplastic administration for previous bone metastasis of medullary thyroid cancer. Pain, bone exposure, and pus discharge in the right mandible and left maxilla were seen. Osteolysis with maxillary cortical bone perforation at the left buccal vestibule, palate, nasal cavity, and maxillary sinus was observed by radiologic images. A biopsy was done at the left maxilla and through pathological evaluation, a parasite with features of pentastome was revealed within the necrotic bone tissue. Further history taking and laboratory evaluation was done. The parasite was suspected to be infected through maxillary open wounds caused by MRONJ. Awareness of intraosseous pentastomiasis should be emphasized not to be missed behind the MRONJ. Proper evaluation and interpretation for past medical history may lead to correct differential diagnosis and therapeutic intervention for parasite infections.
\end{abstract}

Key words: Pentastomida, parasite, osteonecrosis, bisphosphonate-associated osteonecrosis of the jaw (BRONJ)

\section{INTRODUCTION}

Pentastomiasis is a blood sucking, zoonotic parasite infection caused by pentastomes belonging to the phylum Pentastomida. Pentastomes acquired its title for 2 pairs of hooks around the mouth making 5 appendages at the head, which were formerly misunderstood all to be mouths [1]. Although reptiles are the most typical hosts, mammals, including humans, are definite and intermediate hosts in the genus Linguatula $[1,2]$. Human infections have been reported predominantly in the Middle East, Africa, and Southeast Asia [1-5]. Infection is caused by intake of contaminated water sources, vegetation, raw meat of infected intermediate hosts, or close contact with secretion of infected dogs or livestock $[2,6,7]$. Pen-

\footnotetext{
- Received 25 April 2017, revised 16 June 2017, accepted 17 June 2017.

*Corresponding author (khs@yuhs.ac)

(C) 2017, Korean Society for Parasitology and Tropical Medicine

This is an Open Access article distributed under the terms of the Creative Commons

Attribution Non-Commercial License (http://creativecommons.org/licenses/by-nc/4.0) which permits unrestricted non-commercial use, distribution, and reproduction in any

medium, provided the original work is properly cited.
}

tastomes commonly infect the respiratory system, and human soft tissue infections have been noticed at the lung [8,9], nasal cavity [10-12], eye [13-15], oropharynx [4,16], and other viscera $[3,5,6,17,18]$. Unlike soft tissue infection, intraosseous pentastomiasis has not been reported yet.

Here, we report for the first time a case of intraosseous pentastomiasis in the human maxilla suffering from medication related osteonecrosis of the jaw (MRONJ).

\section{CASE REPORT}

A 55-year-old male had continuously visited the Department of Oral and Maxillofacial Surgery at Yonsei University College of Dentistry for MRONJ which had primarily developed after zoledronic acid and capecitabine administration for previous bone metastasis of medullary thyroid cancer. Pain, bone exposure, and pus discharge in the right mandible and left maxilla first occurred 5 years after discontinuing bisphosphonate and capecitabine administration. At that time, the patient was going through chemotherapy with another antineo- 

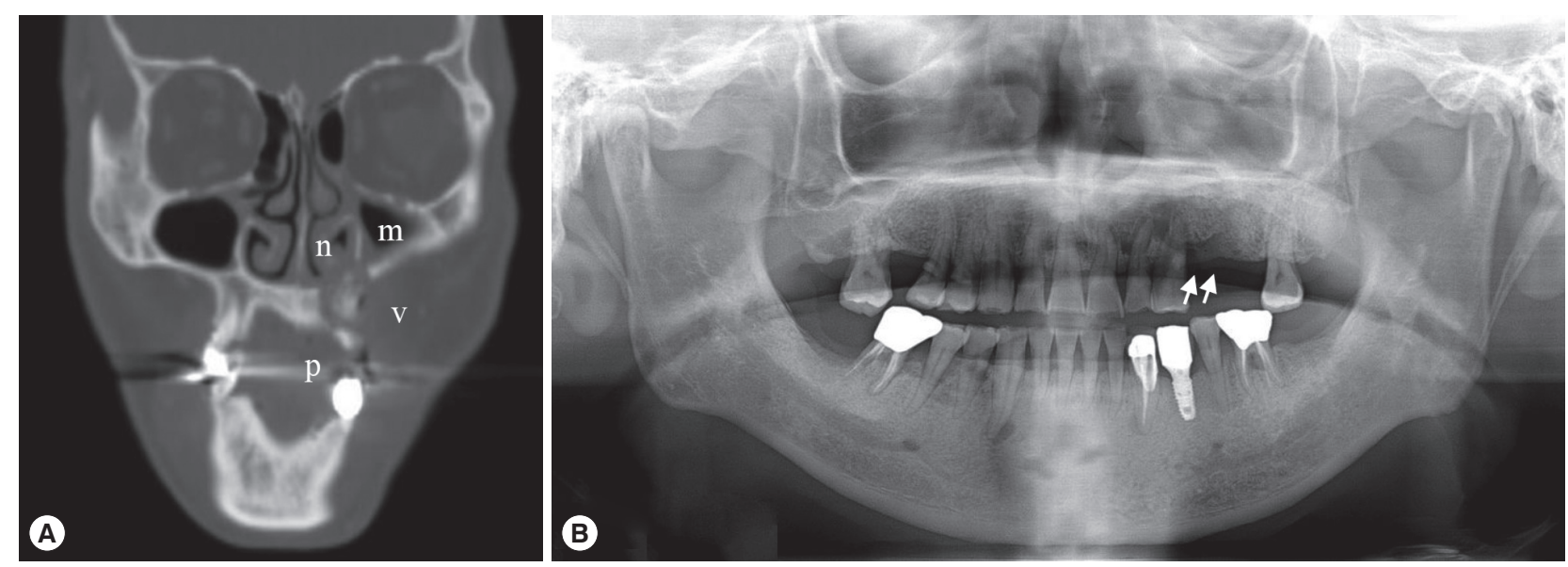

Fig. 1. Radiography of the patient. (A) CT view after primary maxillary bone exposure: osteolytic lesion with sequestra suspected calcification in the left maxilla perforating the cortical bone of the buccal vestibule (v), palate (p), nasal cavity (n), and maxillary sinus (m). (B) Panoramic view of the recurrent maxillary bone exposure with ill-defined radiolucent lesion (arrows) and alveolar bone loss in the left maxilla.

plastic drug, everolimus. Clinical, radiological features of both jaws, and pathological evaluation of the mandible confirmed the diagnosis as MRONJ.

Symptoms of the left maxilla started as chronic apical abscess with tooth mobility in the left premolars. Primary bone exposure with osteonecrosis at the left maxilla was observed after extraction of these teeth in a local clinic. The remaining sockets showed delayed bone healing with pus discharge and incomplete soft tissue covering.

Radiologic examination by CT (Fig. 1A) revealed that there was an irregularly mixed radiolucent/radiopaque area in the left maxilla with discontinuity of the cortical barrier adjacent to the left buccal vestibule, palate, nasal cavity, and maxillary sinus. Mucosal thickening was seen in the left maxillary sinus and nasal cavity. These radiologic features persisted in the additional CT views taken 2 years later (data not shown).

After a year of conservative treatment and another year and a half without periodic follow-up, the patient re-visited the hospital for recurrent bone exposure in the left maxilla with pus discharge. An ill-defined radiolucent/radiopaque lesion with definite alveolar bone loss was seen at the left maxilla on panoramic views (Fig. 1B). The exposed sequestra were removed and sent for pathological evaluation.

On pathological examinations, the tissue specimen revealed mostly several necrotic bone fragments with surrounding nonspecific bacterial colonies and necrotic soft tissue. The bone fragments had mature calcified matrix and irregular margins without any vital osteoblastic or osteoclastic rimming. The lacunae were empty of osteocytes, indicating bone necrosis.
Pathological features were consistent with MRONJ, and there was no evidence of metastatic medullary thyroid cancer. Interestingly, between the bone fragments, there was a wormshaped organism with several false annuli (pseudosegmentation) along the body and features of a hook or claw-like structure at the broader cephalic-end (Fig. 2). Focal areas of necrosis were noticed within the parasite. Due to these features, the organism was diagnosed as a pentastome by further evaluation at the Department of Internal Medicine and the Department of Environmental Medical Biology.

By further oral history taking, the patient disclosed that he had been occupied for about 10 years in foreign trade business since his thirties, with local food exposure in the Middle East, Southeast Asia, and Africa. Short subsequent visits every year continued in several countries. There were no specific symptoms at that time. The patient did not have any specific signs or symptoms of other visceral pentastomiasis. The patient had performed periodic whole blood laboratory tests for thyroid cancer treatment, and the results were evaluated. Right before parasite discovery at the Department of Oral and Maxillofacial Surgery, the laboratory results showed eosinophilia (eosino-

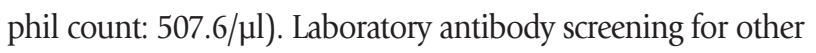
types of parasite infections revealed positive for toxocariasis $\mathrm{IgG}$, and parasiticidal treatments for both pentastomiasis and toxocariasis (albendazole $800 \mathrm{mg} /$ day $\times 5$ days) were prescribed [3]. Since then, periodic follow-up along with supportive care for thyroid cancer is being done. 


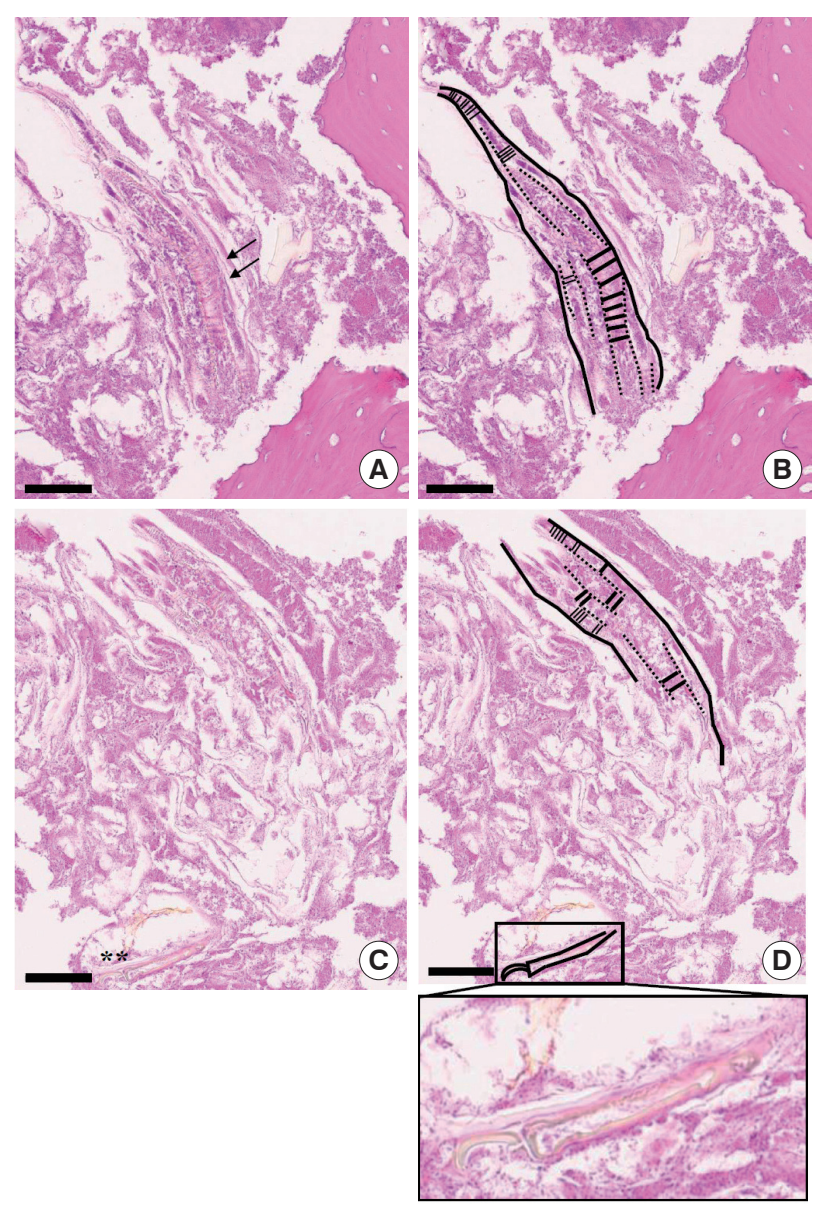

Fig. 2. Pathological findings of the incisional biopsy (scale bar $=60$ $\mu \mathrm{m})$. (A) Histopathological view showing the caudal end of a pentastome with several false annuli (pseudosegmentation; arrows) along the body surrounded by necrotic bone fragments (HE stain, $\times 400$ ). (B) Schematic drawing of Fig. 2A. (C) Serial section of Fig. $2 \mathrm{~A}$ revealing a hook or claw-like structure (asterisks) at the cephalic end (HE stain, $\times 400$ ). (D) Schematic drawing of Fig. 2C. Inset of Fig. 2D shows a more definite image of the hook/claw-like structure of the pentastome.

\section{DISCUSSION}

Pentastomiasis is exceptional in the Republic of Korea with only 1 case of visceral infection reported [8], in which the patient had an occupational history at the Middle East and Southeast Asia. In our case, the remarkable risk factors for pentastomiasis were history of regular cosmopolitan visits to the Middle East, Africa, and Southeast Asia with local food consumption, and most importantly, MRONJ at the maxilla.

The limitations of this case were that an intact worm specimen was not found during the examination, and the parasite infection was only presented through a few sections of histo- pathologic images and indirect evidence of eosinophilia. Whether the sectioned parasite and lesion were an active parasitic infection or embedded traces of a previous infection could not be determined. Serologic tests and PCR techniques for pentastomiasis diagnosis is not easily available with only a few reports of PCR diagnosis in certain Armillifer species $[6,17,19]$. One report discussed that the presence of necrotic tissues in PCR hampers the success rate of molecular diagnosis [19]. Thus, histopathological diagnosis is yet mainly required in pentastomiasis [6]. Our case had only a few sections of available parasite tissue with partial necrosis which was not suitable for PCR and was diagnosed mainly by the histopathological evaluation. The patient did not show any specific signs or symptoms of other visceral infections, but since pentastomiasis can be silent, parasiticidal treatment with albendazole [3] was prescribed.

MRONJ, formerly named as BRONJ (bisphosphonate related osteonecrosis of the jaw) [20], is a clinically obstinate osteonecrotic condition of the jaw characterized by delayed healing, spontaneous bone exposure, pain, inflammation, and/or combined infection [21,22]. It is a problematic side effect which develops in about $0.1-12 \%$ of patients with history of bisphosphonate or antiangiogenic drug administration, and yet the pathogenesis is poorly understood $[22,23]$. In addition to the more well-known MRONJ inducer zoledronic acid $[24,25]$, there has been a few reports suspected of everolimus induced osteonecrosis [26-28]. Microorganism infections are frequently related with MRONJ, and the composition varies among studies $[21,29]$. They were mainly bacterial or fungal species of the normal oral flora that could easily be contaminated through an open wound in the oral cavity $[22,30]$.

Hematogenous migration of pentastome larvae or eggs to the bone may be theoretically possible, as other intraosseous parasites are known to principally infect relatively abundant vascularized sites of the bone, for instance the spine, pelvis, long bone, and major joints [31,32]. However, with the presence of MRONJ in this case, there is a higher probability of the migrants to have directly entered the maxilla via cortical bone perforation from its original habitat, the respiratory tract, oropharyngeal, or nasal cavity.

This is the first case of intraosseous pentastomiasis, as well as the first case of parasitic infection in MRONJ to be reported. Intraosseous echinococcosis, leishmaniasis, and proliferative sparganosis have been mentioned in other sites besides the jaw with/without pathological fracture or trauma [32-35]. 
MRONJ, frequently noticed in the practice of dentistry, is characterized with open wounds prone to infection specifically at the jaw and might create intraosseous chambers of bone marrow infection, including parasitic infection as observed in our case. Intraosseous parasitic infection is uncommon, so routine bone examination during systemic evaluation is not necessary in hosts with known parasite infection. However, if the host has MRONJ, a more detailed examination and pathological evaluation for intraosseous parasitic spread at the jaw might be required.

Although the maxillary defect showed fair healing after removal of the parasite, it is difficult to directly associate the clinical symptoms to intraosseous pentastomiasis with limits of a single case and short follow-up duration. Patients suffering from MRONJ may experience constant switches of osteonecrotic and healing stages [22], making it difficult to judge whether complete treatment of parasite infection was accomplished. If MRONJ is accompanied with specific infections rather than non-specific oral flora, either symptoms of MRONJ or the specific infection (in this case parasitic) may be camouflaged by the other, which may delay proper diagnosis and treatment. Therefore, in cases with prolonged symptoms of MRONJ, further investigation for additional infection sources should be considered.

\section{ACKNOWLEDGMENTS}

This work was supported by grants from the National Research Foundation of Korea (NRF-2017R1C1B1012464, NRF2016R1E1A1A01942724) funded by the Korea government (MSIP) and a grant from the National Research Foundation of Korea (NRF-2014R1A6A3A04055110) funded by the Korea government (MOE).

\section{CONFLICT OF INTEREST}

We have no conflict of interest related to this study.

\section{REFERENCES}

1. Drabick JJ. Pentastomiasis. Rev Infect Dis 1987; 9: 1087-1094.

2. Riley J. The biology of pentastomids. Adv Parasitol 1986; 25: 45128.

3. Vanhecke C, Le-Gall P, Le Breton M, Malvy D. Human pentastomiasis in Sub-Saharan Africa. Med Mal Infect 2016; 46: 269-275.

4. Sulyok M, Rózsa L, Bodó I, Tappe D, Hardi R. Ocular pentasto- miasis in the Democratic Republic of the Congo. PLoS Negl Trop Dis 2014; 8: e3041.

5. Latif B, Omar E, Heo CC, Othman N, Tappe D. Human pentastomiasis caused by Armillifer moniliformis in Malaysian Borneo. Am J Trop Med Hyg 2011; 85: 878-881.

6. Tappe D, Buttner DW. Diagnosis of human visceral pentastomiasis. PLoS Negl Trop Dis 2009; 3: e320.

7. Neva FA, Brown HW. Basic Clinical Parasitology. 6th ed. Norwalk, Connecticut. Appleton \& Lange. 1994.

8. Park CS, Kim MW, Hong WK, Lee HB, Lee DW, Kang DY, Chai JY, Lee SH. Pentastomiasis which caused eosinophilic pneumonia: report of a case. J Korean Med Assoc 1985; 28: 1141-1146.

9. Jisieike-Onuigbo NN, Odenigbo CU, Kalu OA, Eze KC. Armillifer armillatus infection. Niger J Clin Pract 2011; 14: 501-503.

10. Yazdani R, Sharifi I, Bamorovat M, Mohammadi MA. Human linguatulosis caused by Linguatula serrata in the city of Kerman, South-eastern Iran- case report. Iran J Parasitol 2014; 9: 282-285.

11. Papadakis AM, Hourmouziadis AN. Human infestation with Linguatula serrata; report of a case. Trans R Soc Trop Med Hyg 1958; 52: 454-455.

12. Morsy TA, El-Sharkawy IM, Lashin AH. Human nasopharyngeal linguatuliasis (Pentasomida) caused by Linguatula serrata. J Egypt Soc Parasitol 1999; 29: 787-790.

13. Bhende $M$, Biswas J, Raman $M$, Bhende PS. Linguatula serrata in the anterior chamber of the eye. Indian J Ophthalmol 2014; 62: 1159-1161.

14. Koehsler M, Walochnik J, Georgopoulos M, Pruente C, Boeckeler W, Auer H, Barisani-Asenbauer T. Linguatula serrata tongue worm in human eye, Austria. Emerg Infect Dis 2011; 17: 870872.

15. Lazo RF, Hidalgo E, Lazo JE, Bermeo A, Llaguno M, Murillo J, Teixeira VP. Ocular linguatuliasis in Ecuador: case report and morphometric study of the larva of Linguatula serrata. Am J Trop Med Hyg 1999; 60: 405-409.

16. Yilmaz H, Cengiz ZT, Cicek M, Dulger AC. A nasopharyngeal human infestation caused by Linguatula serrata nymphs in Van province: a case report. Turkiye Parazitol Derg 2011; 35: 47-49.

17. Tappe D, Dijkmans AC, Brienen EA, Dijkmans BA, Ruhe IM, Netten MC, van Lieshout L. Imported Armillifer pentastomiasis: report of a symptomatic infection in The Netherlands and minireview. Travel Med Infect Dis 2014; 12: 129-133.

18. Wang HY, Zhu GH, Luo SS, Jiang KW. Childhood pentastomiasis: a report of three cases with the following-up data. Parasitol Int 2013; 62: 289-292.

19. Tappe D, Sulyok M, Rozsa L, Muntau B, Haeupler A, Bodo I, Hardi R. Molecular diagnosis of abdominal Armillifer grandis pentastomiasis in the Democratic Republic of Congo. J Clin Microbiol 2015; 53: 2362-2364.

20. Ruggiero SL, Dodson TB, Fantasia J, Goodday R, Aghaloo T, Mehrotra B, O'Ryan F. American Association of Oral and Maxillofacial Surgeons position paper on medication-related osteonecrosis of the jaw--2014 update. J Oral Maxillofac Surg 2014; 72: 1938-1956. 
21. Boff RC, Salum FG, Figueiredo MA, Cherubini K. Important aspects regarding the role of microorganisms in bisphosphonaterelated osteonecrosis of the jaws. Arch Oral Biol 2014; 59: 790799.

22. Ruggiero SL, Woo SB. Biophosphonate-related osteonecrosis of the jaws. Dent Clin North Am 2008; 52: 111-128.

23. Lombard T, Neirinckx V, Rogister B, Gilon Y, Wislet S. Medication-related osteonecrosis of the jaw: new insights into molecular mechanisms and cellular therapeutic approaches. Stem Cells Int 2016; 2016: 8768162

24. Bamias A, Kastritis E, Bamia C, Moulopoulos LA, Melakopoulos I, Bozas G, Koutsoukou V, Gika D, Anagnostopoulos A, Papadimitriou C, Terpos E, Dimopoulos MA. Osteonecrosis of the jaw in cancer after treatment with bisphosphonates: incidence and risk factors. J Clin Oncol 2005; 23: 8580-8587.

25. Cetiner S, Sucak GT, Kahraman SA, Aki SZ, Kocakahyaoglu B, Gultekin SE, Cetiner M, Haznedar R. Osteonecrosis of the jaw in patients with multiple myeloma treated with zoledronic acid. J Bone Miner Metab 2009; 27: 435-443.

26. Fusco V, Santini D, Armento G, Tonini G, Campisi G. Osteonecrosis of jaw beyond antiresorptive (bone-targeted) agents: new horizons in oncology. Expert Opin Drug Saf 2016; 15: 925-935.

27. Giancola F, Campisi G, Lo Russo L, Muzio LL, Di Fede O. Osteonecrosis of the jaw related to everolimus and bisphosphonate: a unique case report? Ann Stomatol (Roma) 2013; 4 (suppl): 2021.
28. Kim DW, Jung YS, Park HS, Jung HD. Osteonecrosis of the jaw related to everolimus: a case report. Br J Oral Maxillofac Surg 2013; 51: e302-304.

29. Wei X, Pushalkar S, Estilo C, Wong C, Farooki A, Fornier M, Bohle G, Huryn J, Li Y, Doty S, Saxena D. Molecular profiling of oral microbiota in jawbone samples of bisphosphonate-related osteonecrosis of the jaw. Oral Dis 2012; 18: 602-612.

30. Sedghizadeh PP, Kumar SK, Gorur A, Schaudinn C, Shuler CF, Costerton JW. Microbial biofilms in osteomyelitis of the jaw and osteonecrosis of the jaw secondary to bisphosphonate therapy. J Am Dent Assoc 2009; 140: 1259-1265.

31. Arkun R. Parasitic and fungal disease of bones and joints. Semin Musculoskelet Radiol 2004; 8: 231-242.

32. Abd El Bagi ME, Sammak BM, Al Shahed MS, Yousef BA, Demuren OA, Al Jared M, Thagafi MA. Rare bone infections "excluding the spine". Eur Radiol 1999; 9: 1078-1087.

33. Laovachirasuwan P, Mootsikapun P, Maleewong W, Intapan PM. Intraosseous proliferative sparganosis presenting as a pathologic fracture: A case report and review of the literature. Southeast Asian J Trop Med Public Health 2015; 46: 558-563.

34. Rajabi MT, Akbari-Kamrani M, Gharib MH, Rajabi MB, Amoli FA, Abrishami Y, Oestreicher J. Intraosseous orbital hydatid cyst: report of a rare case. Can J Ophthalmol 2014; 49: e40-43.

35. Arik HO, Arican M, Cetin NK, Sarp U. Primary intraosseous hydatid cyst of femur. Iran Red Crescent Med J 2015; 17: e21070. 
